EDITORIAL

\title{
An overview of Portugal's educational challenges and responses to the global pandemic of COVID-19
}

\author{
Filipa Alves da Costa ${ }^{1,2,3}$ (D), Maria Deolinda Auxtero ${ }^{3}$, Ana Paula Martins ${ }^{1,2,4}$, Maria Margarida Caramona ${ }^{2,5}$ \\ ${ }^{1}$ Department of Social Pharmacy, Faculty of Pharmacy, University of Lisbon, Portugal \\ ${ }^{2}$ Portuguese Pharmaceutical Society (Ordem dos Farmacêuticos), Lisbon, Portugal \\ ${ }^{3}$ CiiEM, Interdisciplinary Research Center Egas Moniz, Instituto Universitário Egas Moniz, Quinta da Granja, Caparica, Portugal \\ ${ }^{4}$ Institute for Evidence-Based Health, Faculty of Medicine, University of Lisbon, Portugal, Lisbon, Portugal \\ ${ }^{5}$ Faculty of Pharmacy, University of Coimbra, Coimbra, Portugal
}

\section{Correspondence}

Filipa Alves da Costa

Department of Social Pharmacy

Faculty of Pharmacy

University of Lisbon

Av. Prof. Gama Pinto

1649-003 Lisboa

Portugal

alvesdacosta.f@gmail.com

\begin{abstract}
The current pandemic of SARS-CoV-2 came un-expectantly and required unprecedented ability to adapt and quickly put in place the most appropriate response measures to limit the spread of COVID-19 disease, while minimising disruption to society's essential activities. Some sectors were more capable of adapting and reacting than others. Higher education was one of the sectors where the academic capacity (FIP Development Goal 1) demonstrated was phenomenal, partially attributable to the ability to create partnerships between academia and practice. This editorial provides a description of the major changes put in place in Portugal to ensure the education and training of future graduates in Pharmaceutical Sciences was maintained with the same quality. It also discusses some of the potentially less beneficial long-term impacts of these adaptions to the teaching and assessment methods for the competencies of the workforce of tomorrow.
\end{abstract}

An overview of Portugal's educational challenges and responses to the global pandemic of COVID-19

\section{Education in Pharmaceutical Sciences in Portugal}

In Portugal there are nine institutions providing education in pharmaceutical sciences, all of them offering an Integrated Masters, with a duration of four and a half years. There is also a demand to undergo an internship period of 22 weeks during or after the formal theoretical and practical training acquired in the campus, which may be pursued either in community or hospital pharmacy. The European Credit Transfer and Accumulation System (ECTS) is used, through which the entire degree corresponds to 300 ECTS (one ECTS credit corresponds to 25-30 hours of study; internship corresponds to 30 ECTS). All these aspects are aligned with the European directive, and thus enable student and professional mobility within the European space (European Parliament and of the Council, 2013). Even though there is diversity and flexibility in the curricular units offered by the different academic institutions, they all follow this directive and their students are therefore recognised by the professional body as fit for practice.

\section{Traditional educational methods in Portugal}

There is diversity in teaching methods employed. However, according to the European Pharmacy Students Association, there is room for improvement in one of the four pillars considered crucial for successful pharmacy education, by enhancing opportunities for experiential and hands-on learning (European Pharmaceutical 
Students' Association, 2018). During the peak of the pandemic, FIP conducted a rapid response survey targeted at Global Higher Education in Pharmacy that aimed to capture the immediate impact of shifting pharmacy education and training to online remote delivery models as a result of SARS-CoV-2. Most faculties in Portugal responded, coordinated by the Education and Practice Platform, a consultant body within the Portuguese Pharmaceutical Society (Alves da Costa, et al., 2020). Data obtained overall clearly demonstrates that, with some exceptions, most institutions did not previously routinely use online teaching methods, including seminars, one-to-one tutorials and student discussion groups. Conversely, most faculties were already resorting to online module reading materials, often made available through institutional or internal virtual learning environments (e.g. Moodle). There are also reports of the use of mobile applications to engage students in learning before the pandemic.

\section{Changes in educational methods during SARS-CoV2 pandemic}

SARS-CoV-2 causing COVID-19 disease, arose in Wuhan on the 31st of December 2019. One month later, on 30th January, the World Health Organisation (WHO) declared COVID-19 a Public Health Emergency of International Concern and on the 11th of March classified it as a pandemic, given the likelihood of further spread, potential impact on human health and effectiveness of preparedness and response measures in place. All pharmacy education institutions in Portugal adopted a decision as a response to the COVID-19 crisis between the 8th and the 12th of March, so in some cases, even ahead of the WHO communication, clearly highlighting an anticipatory strategy.This decision had some variation, with the majority opting for immediate closure and a switch in education to remote/online delivery, while a few institutions located in less affected areas of the country maintained face-to-face learning for a limited period during which the transition of remote delivery was progressively made. In a period of one-to-two weeks, all institutions were offering their students a mix between pre-recorded online lectures delivered in asynchronous online format and synchronous lectures, i.e., delivered in a chosen time by students or in real time, respectively. While the first was not new to many, synchronous teaching was innovative for eight out of the nine institutions, revealing an enormous capacity to adapt to adversity. It also highlights how academic capacity, as defined by FIP Development Goal 1 (DG Workforce) adapted education and training programmes fit for purpose according to the emerging needs to provide a competent pharmaceutical workforce.

\section{Internships in community and hospital pharmacy}

Internships are an extremely important part of any degree, as they correspond to the stage where the student should be able to do. This is the top of Miller's pyramid, so while it was important to maintain the ability of students to 'know' and to 'know how' for those in the first cycle of the integrated masters, these aspects refer to cognitions, and are therefore relatively easier to adapt to the technological challenges. Cognitions do not necessarily translate into behaviours, thus not ensuring a competent workforce. For cognitions to be translated into behaviours, the students must be able to 'show how' to do it, something also possible to resort to remote learning by using clinical case assignments or similar methods. However, being able to 'do' is only possible by enabling students to practice in a real-life environment. The European Directive states that this phase may be conducted in community or in hospital pharmacy, which implies that faculties can adapt their offer according to the students' preferences and the placements available, i.e., offer both or one of these areas. During the lockdown period, practices were not open to receive interns for safety reasons and therefore internships were generally postponed. Academia extended their evaluation periods so that students were not jeopardised by the pandemics, while ensuring all students had the opportunity to have their internship guaranteed. Simultaneously, an agreement was established between sectoral organisations; the pharmaceutical society and all faculties were to offer free of charge to all students the opportunity to complement their training with access to an online platform created by professionals for professionals, the FIT programme. The acronym chosen results from the motto: Train people, Innovate in the concept and Transform the learning experience. This programme is organised to facilitate the acquisition of competences in four major domains: scientific-technical, operational efficiency, technological and behavioural. All institutions were offered this opportunity and were free to include it as compulsory or optional training for their students, allowing flexibility in the evaluation methods. This initiative highlights the ability to create a purposeful training pathway to support post-registration foundation training, aligned with FIP DG2 (Workforce). 


\section{Challenges}

The adaption to educational methods resorting to a constant use of the internet has many advantages but also challenges which may result in inequities in access to education. On the one hand, during this period students had the possibility to go back to their homes, which diminished difficulties associated to geographic location and socioeconomic status to enable students to live on their own or on school campuses. Conversely, going back to their living accommodation also led to some difficulties in accessing the internet, as the wide band coverage is not universal throughout the country and is affected by the simultaneous number of users, leaving students from rural areas or living in households with various family members working and studying simultaneously in difficult conditions.

The second challenge, which was cross-sectional to all students, was the inability to maintain personal contact, value group work and the human component involved in such interactions. This has a potential impact on the future workforce's ability to work in inter-professional and interdisciplinary teams and to efficiently interact with others.

\section{Case studies}

As in all professions, there are pharmacy professors truly pedagogical in their approach and fully dedicated to make students gain knowledge and skills. It would be impossible to describe all interesting and unusual cases of devoted professors taking a unique approach to the pandemic challenge. Laboratory courses were the ones more severely hit by the impossibility of physical presence, among which galenic pharmacy is included. This curricular unit aims at producing pharmaceutical formulas, emphasising fundamental unitary operations, including milling, sieving, suspension, simple and extractive dissolution, coarse division by section, among others.

Figure 1 illustrates a home-made class in a professor's kitchen that was transformed into a learning environment by replacing the traditional raw materials and active ingredients by nutritional ingredients, including flower, sucrose and aromatic plants. Pharmaceutical formulation available at home have also been used to demonstrate the essential techniques for drug development. The demonstration by the professor was videoed and made available to students asynchronously, who during synchronous class could reproduce the experience in their own homes supervised by the teacher. As a complement, quizzes were used to evaluate the understanding of techniques used.

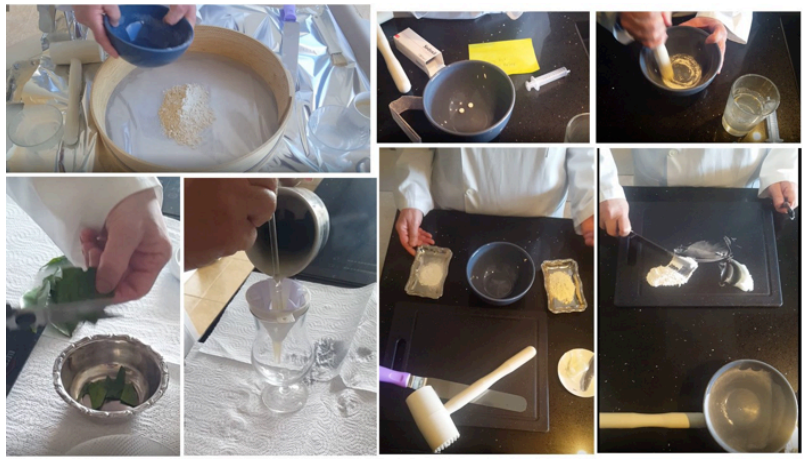

\section{Success factors}

There were three main vectors for the successful adaption of pharmacy education. The first resulted from previously existing digital platforms that enabled remote learning and teaching, which were in place ahead of the pandemics, mostly for theory-based lectures, which were quickly maximised to also include more practical teaching methods and combined with other resources to enable synchronous learning (e.g. MS Teams, Zoom, Google classrooms, etc.).

There was a similar situation of disruption in Portugal during the revolution in 1974, and at the time, many students had administrative approvals. In the current pandemics, academia was able to maintain the evaluation rigour, resorting above all to online timed multiple-choice questions (MCQ) exams, which were programmed to randomly shuffle questions and allow enough variability in questions used. Other used methods included online written short answer exam, online open book examinations, submission of course work, online experiential learning workbooks and portfolios and to a lesser extent online OSCE-style examinations or application-based assessments. All these enabled meeting the educational objectives, including knowledge acquisition and demonstration.

The third success factor was the creation of an open platform for dialogue between all involved stakeholders. There was constant promotion of discussion between students' associations, academia, sectoral associations and the Educational and Practice platform to overcome barriers and find possible solutions to ensure the quality of education was maintained and identify the necessary adaptions needed to meet the common interests of all. The Educational and Practice platform was created to bridge the distance between academics and practitioners and at the time its usefulness for managing crisis was certainly not foreseen but proved to be valuable in the context of SARS-CoV-2 pandemics. 


\section{Future perspectives}

Crises often result in opportunities and for the current curricular year, 2020/2021, academia had more time to further improve their teaching and evaluation methods and learn from their experiences. There is a move now to convert e-learning into blended-learning approaches, so that face-to-face contact is maintained allowing for interpersonal competences to be developed, while maximising response measures put in place to prevent and control a possibly arising new wave of infection. The transformation of syllabus into modular options is also being adopted whenever possible to allow enough flexibility for students to meet their learning needs while enabling the possibility to maintain laboratory and clinical components onsite.

\section{Acknowledgements}

To the five institutions who voluntarily participated in the FIP survey entitled 'Global Higher Education in Pharmacyresponding to COVID-19', including Universidade da Beira Interior, Universidade Lusófona de Humanidades e Tecnologias- Escola de Ciências e Tecnologias da Saúde, Instituto Universitário Egas Moniz, Escola Superior de Saúde da Universidade do Algarve, and Instituto Universitário de Ciências da Saúde (IUCS). To the professors who provided valuable information from the remaining faculties, Matilde Castro (Faculty of Pharmacy, University of Lisbon), Isabel Vitória (Faculty of Pharmacy, University of Coimbra) and Domingos Ferreira (Faculty of Pharmacy, University of Porto).

\section{References}

Alves da Costa, F., Martins, A., Veiga, F., Ramalhinho, I., Lobo, J., Rodrigues, L., Granadeiro, L., Castro, M., Barata, P., Gomes, P., Seabra, V., \& Caramona, M. (2020). Development of a Platform to Align Education and Practice: Bridging Academia and the Profession in Portugal. Pharmacy, 8(1), 11. https://doi.org/ 10.3390.pharmacy8010011

European Parliament and of the Council. (2013). Directive 2013/55/EU of 20 November, amending Directive 2005/36/EC and Regulation (EU) No 1024/2012. Brussels. Available at: https://eur-lex.europa.eu/legal-content/EN/TXT/PDF/?uri=CELEX: 32013L0055\&from=PT

European Pharmaceutical Students' Association. (2018). The Methodology Booklet. . (E. Commission, Ed.) Brussels. Available at: https://www.epsa-online.org/methodology-booklet/ 